\title{
Direct Handling of Sharp Interfacial Energy for Microstructural Evolution
}

\author{
Efraín Hernández-Rivera ${ }^{\mathrm{a}, \mathrm{b}}$, Veena Tikare ${ }^{\mathrm{b}, *}$, Laurence Noirot ${ }^{\mathrm{c}}$, Lumin \\ Wanga \\ ${ }^{a}$ University of Michigan, Department of Nuclear Engineering and Radiological Sciences, \\ Ann Arbor, Michigan 48109, USA \\ ${ }^{b}$ Sandia National Laboratories, Advanced Nuclear Energy Programs Department, \\ Albuquerque New Mexico 87185, USA \\ ${ }^{c}$ Commissariat à l'Énergie Atomique Cadarache, Saint-Paul lez Durance, \\ Provence-Alpes-Côte-d'Azur 13108, FR
}

\begin{abstract}
We introduce a simplification to the previously demonstrated hybrid Pottsphase field (hPPF), which relates interfacial energies to microstructural sharp interfaces. The model defines interfacial energy by a Potts-like discrete interface approach of counting unlike neighbors, which we use to compute local curvature. The model is compared to the hPPF by studying interfacial characteristics and grain growth behavior. The models give virtually identical results, while the new model allows the simulator a more direct approach to model interfacial energy.
\end{abstract}

Keywords: Cahn-Hilliard, Curvature driven flux, Sharp interface, Potts Monte Carlo

Many microstructural evolution processes have been simulated by the Cahn-Hilliard $(\mathrm{CH})$ based phase field model. The Cahn-Hilliard equa-

\footnotetext{
*Corresponding author

Email address: vtikare@sandia.gov (Veena Tikare)
} 
tion was originally developed to model spinodal decomposition [1] of glass with interfacial energy introduced by a gradient in the composition of the glass. Since then, many have adapted this to simulate a wide range of microstructural evolution mechanisms with "order parameters" introduced by Khachaturyan [2] to represent many other materials characteristics, such as grains and precipitates with a gradient in the parameters representing the interfaces. Recently, Homer et. al. [3, 4, 5] introduced a hybrid Potts-Phase field (hPPF) model, which uses both the Cahn-Hilliard (CH) smoothly varying interfacial energy and the Potts sharp interfacial energy terms to calculate the interfacial energy. In this work, we show that the $\mathrm{CH}$-interfacial energy term can be eliminated; we can determine the interfacial energy of sharp interfaces between discrete particle and the bulk free energy by the compositional field. We will compare our results to those of the hPPF model, which has been extensively studied. The benefit of such a model is that it affords direct control over the thermodynamics of interfaces. These interfaces between microstructural features are sharp interfaces with the associated interfacial energy, $\gamma S$, which makes the bulk energy equation a function of phase and composition.

Several types of computational techniques have been used to model the evolution of microstructures, e.g. Potts Monte Carlo (PMC) and phase field $(\mathrm{PF})$ models. The former uses a discretized ensemble of particles, while the latter uses continuum fields, or order parameters, to define the microstructure. Hence, the PMC and PF models have sharp and diffuse interfaces, respectively. While these models have been extensively used for different types of microstructural evolution, both have intrinsic drawbacks. The PMC 
model struggles simulating smoothly varying continuous fields like concentrations. While the PF model requires a large set of coupled partial differential equations with meshes sufficiently refined to accurately capture diffuse interface evolution. These issues can easily become prohibitively computationally expensive. Therefore, Homer et. al. developed the hPPF model that enables efficient and accurate simulation of microstructural evolution where microstructure is characterized by both continuous fields and discrete particles.

Many applications of the PF model uses the Cahn-Hilliard equation [1, 6, 7, 8], where the interfacial energy is a function of the gradients in the continuous fields. The hybrid model developed by Homer et al., incorporates both this interfacial energy as well as the Potts interfacial energy. In this work, we implement a simpler, yet robust, approach to calculating the interfacial energy with more direct control over it's characteristics. In essence, our model uses a discretized curvature, where the digitized microstructural features are used to calculate the interfacial energy, as opposed to gradients on the concentration field.

Our model runs in a digitized voxelated mesh with a continuum concentration field overlaid on it. The voxels are populated by an ensemble representing the different microstructural features. The microstructure is represented by a set of discrete fields, grain id $(s)$ and phase $(q)$, and a continuum field, concentration $(C)$. The concentration, $C \in \mathbb{R}: 0 \leq C \leq 1$, defines the fraction of component $B$ at a point in the continuum field. The grain id and phase are integer discrete values that denote the membership of that voxel in a particular grain and its phase, respectively. For the grain 
growth simulation, we have a two-phase binary system where each phase is allowed a set of grain ids.

The thermodynamic state of the system is given by an equation of state (EoS), which has volumetric and interfacial terms. The hPPF model uses a free energy functional that incorporates the gradient in composition term used by the $\mathrm{CH}$ equation and the Potts term, i.e. total number of dissimilar neighboring voxels,

$$
F=\int_{\mathcal{V}} f_{0} d V+\int_{\mathcal{S}} \gamma d S=\int_{\mathcal{V}} f_{0} d V+\frac{\gamma_{C H}}{2} \int_{\mathcal{V}}|\nabla C|^{2} d V+\gamma_{\text {Potts }} \int_{\mathcal{S}} d S
$$

where $f_{0}$ is the bulk chemical free energy and the $\gamma$ 's are the interfacial or surface energy. The $\mathrm{CH}$ term goes to zero as we move away from the interface

$$
\lim _{d_{\text {int } \rightarrow \infty}} \nabla C=0
$$

where $d_{\text {int }}$ is the distance to the sharp interface. Our model only uses the Potts term to account for the interfacial energy induced by the curved surface (curvature), which gives us

$$
F=\int_{\mathcal{V}} f_{0} d V+\gamma_{\text {Potts }} \int_{\mathcal{S}} d S
$$

We use polynomial (quadratic) equations to describe the bulk chemical free energies

$$
f=\int f_{0} d V=\sum_{i=1}^{N} f_{i}\left(q_{i}\right)
$$

where $N$ is the total number of sites (voxels) in the simulation system and each site $i$ with $q$-phase stores energy

$$
f_{i}= \begin{cases}\lambda_{0}\left[\left(C-C_{1}\right)^{2}+\left(C-C_{2}\right)^{2}\right]+\lambda_{1}\left(C-C_{3}\right)^{2}, & q=\alpha \\ \lambda_{0}\left[\left(C-C_{1}\right)^{2}+\left(C-C_{2}\right)^{2}\right]+\lambda_{1}\left(C-C_{4}\right)^{2}, & q=\beta\end{cases}
$$


where $f_{i}$ is the bulk free energy for the $\alpha$ - and $\beta$-phases, and the $\lambda$ 's and $C_{i}$ 's constants are the parameterization variables chosen to match the work by Homer, Table 1 .

The general microstructural evolution for multi-state systems has been simulated by the Potts Monte Carlo model [9], which we incorporate to calculate the sharp interfacial energy, given by

$$
\int_{\mathcal{S}} \gamma_{P o t t s} d S \equiv J \sum_{i=1}^{N} \sum_{j=1}^{n}\left(1-\delta_{s_{i} s_{j}}\right)
$$

where $J$ is the interaction energy between adjacent elements, $n$ is the number of neighboring particles, $\delta$ is the Kronecker delta, and $s_{i}$ and $s_{j}$ are the grain id for the $i$ and $j$ sites. Then, introducing Equations 5 and 6 to Equations 1 and 3, we get the following discretized equations of state

$$
\begin{gathered}
F_{C H, i}=\sum_{i=1}^{N}\left(f_{0}\left(q_{i}, C_{i}\right)+\frac{\gamma_{C H}}{2}\left|\nabla C_{i}\right|^{2}+J \sum_{j=1}^{n}\left(1-\delta_{s_{i} s_{j}}\right)\right) \\
F_{\text {Pott }, i}=\sum_{i=1}^{N}\left(f_{0}\left(q_{i}, C_{i}\right)+J \sum_{j=1}^{n}\left(1-\delta_{s_{i} s_{j}}\right)\right) .
\end{gathered}
$$

To simulate grain growth and phase coarsening, we minimize the EoS by means of the standard Metropolis algorithm. For each site $i$, we choose a neighboring, site $j$ at random and attempt to adopt the neighbor's id and phase. The change is adopted following Boltzmann statistics, the probability of accepting the microstructural change is given by

$$
P_{i}= \begin{cases}1, & \Delta F_{i} \leq 0 \\ \exp \left(-\frac{\Delta F_{i}}{k_{B} T}\right), & \Delta F_{i}>0\end{cases}
$$


where $\Delta F_{i}$ is the change of energy for the event calculated using Equations 7 or $8, k_{B}$ is the Boltzmann constant and $T$ is the absolute simulation temperature. For the concentration evolution, we use the phenomenological equation

$$
\frac{\partial C}{\partial t}=\nabla \cdot(M \nabla \mu)
$$

where $\mu$ is the chemical potential and $M$ is the mobility. The chemical potential is defined as $\mu \equiv \delta F / \delta C$. Incorporating Equations 1 and 3 into Equation 10 and non-dimensionalizing, for the hPPF model we get

$$
\frac{\partial C}{\partial \tilde{t}}=\tilde{\nabla} \cdot\left[\tilde{\nabla}\left(\frac{\partial \tilde{f}_{0}}{\partial C}-\tilde{\nabla}^{2} C\right)\right]
$$

where $\tilde{t}, \tilde{\nabla}$ and $\tilde{f}_{0}$ are the non-dimensionalized time, spatial gradient and bulk chemical free energy, respectively. For our sharp interface model we get

$$
\frac{\partial C}{\partial \tilde{t}}=\tilde{\nabla} \cdot\left[\tilde{\nabla}\left(\frac{\partial \tilde{f}_{0}}{\partial C}+\tilde{\kappa}\right)\right]
$$

where $\tilde{\kappa}=\gamma_{P o t t s} \Omega \kappa$ is the non-dimensionalized surface curvature 1 . The Potts interfacial energy, $\gamma_{\text {Potts }}$, was chosen to match the $\mathrm{CH}$ interfacial energy, $\gamma_{C H}$, used in the hPPF model.

\footnotetext{
${ }^{1}$ If the energy associated to a curved surface is given by $F=\gamma S$, the variational energy is given by

$$
\frac{\delta F}{\delta C}=\gamma \frac{\delta S}{\delta C}
$$

Adding mass to the surface leads to a change in volume, $\delta V=4 \pi R^{2} \delta r$, and in surface area, $\delta S=8 \pi R \delta r$. Defining the amount of volume added per change in concentration as the molar volume, $\delta V / \delta C=\Omega$, and applying the chain rule, we have

$$
\frac{\delta F}{\delta C}=\gamma \frac{\delta S}{\delta C}=\gamma \frac{\delta V}{\delta C} \frac{\delta S}{\delta V}=\gamma \Omega \frac{8 \pi R \delta r}{4 \pi R^{2} \delta r}=\frac{2 \gamma \Omega}{R} \equiv \gamma \Omega \kappa .
$$
}


Curvature of a continuous field is commonly defined as the divergence of the of the unit normal to the surface, $\kappa=\nabla \cdot \bar{n}$, where $\bar{n}=\nabla C /|\nabla C|$. If $\nabla C$ is nearly constant, we get $\kappa=\nabla^{2} C$, which is consistent with the common curvature definition. We define a discrete curvature that is given directly by the digitized microstructural features. The discrete curvature is defined as

$$
\kappa_{i}=\frac{\sum_{j=1}^{n_{s}}\left(1-\delta_{q_{i} q_{j}}\right)-n_{\text {base }}}{n_{s}}
$$

where $n_{s}$ is the number of surrounding neighbors being considered on the curvature calculation ${ }^{2}, q_{i}$ and $q_{j}$ are the phase at site $i$ and neighbor $j$, respectively, and $n_{\text {base }}$ is the reference state that yields a curvature of zero for a flat surface

$$
n_{\text {base }}=\mathcal{N}(1+2 \mathcal{N})^{D-1}
$$

where $\mathcal{N}$ is the number of neighbors "shells" and $D$ is the simulation dimension. Similar definitions for the discretized curvature have been extensively used by Potts Monte Carlo simulations. Neighboring shells, $\mathcal{N}$, denote the number of neighbors of site $i$ that are used to determine curvature. For $\mathcal{N}=1$, one layer of sites surrounding site $i, n_{s}$ is 8 in 2D and 26 in 3D. Increasing the number of shells yields a more sensitive curvature approximation since more neighboring sites are being considered, making different surface features easier to distinguish. On the other hand, the computational efficiency suffers as the curvature calculation function needs to examine rapidly

\footnotetext{
${ }^{2}$ The number of sites accounted for the curvature calculation can be computed by

$$
n_{s}=(1+2 \mathcal{N})^{D}-1
$$
}


increasing number of neighbors.

The first test compares our model to the hPPF by allowing a $\beta$-precipitate in an $\alpha$-matrix to equilibrate in composition and size. The resulting concentration profiles for the hPPF and $\mathcal{N}=1,2$ and 3 models at $k_{B} T=2 J$ are shown in Figure 1 for when equilibrium is reached, characterized by steady state conditions. The discretized curvature is able to accurately simulate the compositional evolution and is comparable to the hPPF model. The presence of sharp interfaces can be clearly seen in the region around the $\alpha-\beta$ interface. Our model is able to accurately simulate the evolution of the system as the system approaches equilibrium.

We performed a more complex simulation of grain growth controlled by diffusion-limited kinetics in order to verify that the model maintains it's accuracy as the microstructural evolution scales in complexity. The simulations were run at a finite temperature, $k_{B} T=0.3 J$ to avoid grain growth pinning effects. The microstructural evolution can be easily appreciated from Figure 2 where we illustrate both grain growth and compositional evolution. The grain growth curves obtained from the hPPF model are compared to those from our model for shells $\mathcal{N}=1$ and 2 in Figure 3. The grain growth is virtually identical for all three cases. By fitting the data to the grain growth equation, $\bar{R}=\left(m t+R_{0}^{n}\right)^{1 / n}$, we obtained a grain growth exponent of $n=3$ for all three simulations, as shown in Table 2. This grain growth exponent $n=3$ is characteristic of diffusion-controlled Ostwald ripeninglike grain growth kinetics [4, 10, 11]. Since $\mathcal{N}=1$ and $\mathcal{N}=2$ give virtually identical results, the additional computational expense of considering more neighbors for the curvature calculations is not necessary. 
Finally, we simulated the evolution of a polycrystalline material in a 3D system. Figure 4 shows the concentration and microstructural (grain id) evolution of an initially randomly oriented microstructure. The grain growth exponent for the simulation is that predicted by grain growth for a diffusionlimited case, $n=3$. It is evident that our simplified method is able to simulate this type of realistic microstructural evolution while preserving the correct physics and kinetics of the system.

\section{Conclusion}

We have shown that using the discretized curvature approach with continuum thermodynamic description of the bulk energy is accurate and robust for simulation of microstructural evolution. It has been successfully applied to continuum thermodynamic and kinetic equations to simulate microstructural evolution by capillarity. More importantly, we showed that the discretized curvature approach grants us a direct method for calculating the thermodynamic state, rather than using the $\mathrm{CH}$, which was originally developed for spinodal decomposition. Lastly, we showed that the model is robust and can be extended to a 3D scheme and more complex microstructural evolution scenarios.

\section{Acknowledgement}

Sandia National Laboratories is a multi-program laboratory managed and operated by Sandia Corporation, a wholly owned subsidiary of Lockheed Martin Corporation, for the U.S. Department of Energy's National Nuclear 
Security Administration under contract DE-AC04-94AL85000.

[1] J.W. Cahn, Acta Met., 9 (1961) 795.

[2] A.G. Khachaturyan, The Theory of Structural Transformations in Solids, Wiley \& Sons, New York (1983)

[3] V, Tikare, E.R. Homer and E.A. Holm, Cer. Mats. Energy Apps. II: Cer. Eng. and Sci. Procs., (2012) 145.

[4] E.R. Homer, V. Tikare and E.A. Holm, Compt. Mat. Sci., 69 (2013) 414.

[5] J.J. Cox, E.R. Homer and V. Tikare, MRS 2012 Procs., (2013) 1524.

[6] J.W. Cahn and J.E. Hilliard, J. Chem. Phys., 28 (1958) 258.

[7] J.W. Cahn, J. Chem. Phys., 30 (1959) 1121.

[8] J.W. Cahn and J.E. Hilliard, J. Chem. Phys., 31 (1959) 688.

[9] E.A. Holm and C.C. Battaile, JOM, 53 (2001) 20.

[10] I.M. Lifshitz and V.V. Slyozov, J. Phys. Chem. Sol., 19 is. 1-2 (1961) 35.

[11] C. Wagner Zeitschrift für Elektrochemie 65 is. 7 (1961) 581. 
Table 1: Numerical values of the model's parameterization variables used in the presented simulations.

\begin{tabular}{|l|cccccc|}
\hline Parameterization & $C_{1}$ & $C_{2}$ & $C_{3}$ & $C_{4}$ & $\lambda_{0}$ & $\lambda_{1}$ \\
Variable & & & & & & \\
\hline Values & 0.25 & 0.75 & 0.05 & 0.95 & 0.3 & 0.5 \\
\hline
\end{tabular}

Table 2: Summary of the grain growth exponential inverse, $1 / n$, for the $\mathrm{hPPF}$ model and a series of discrete curvature calculations with varying $\mathcal{N}$.

\begin{tabular}{|c|cc|c|}
\hline hPPF & \multicolumn{2}{|c|}{$\mathcal{N}$} & $\mathcal{N}_{3 D}=1$ \\
\hline 0.350 & 1 & 2 & \\
\hline
\end{tabular}




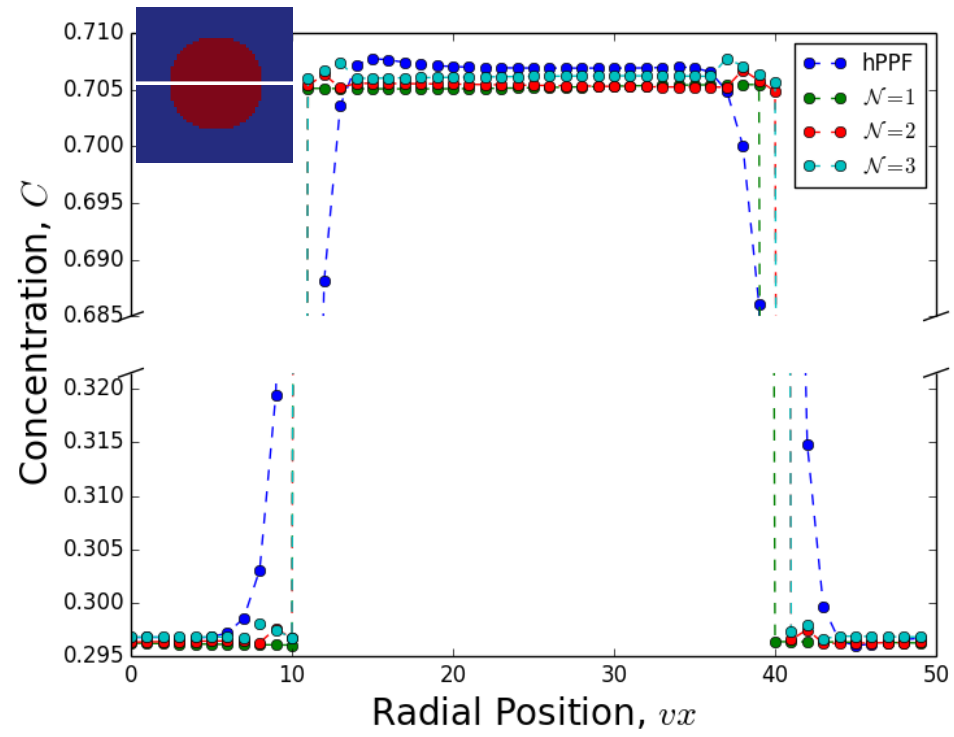

Figure 1: Concentration profile for the matrix-precipitate (insert) system after quasiequilibrium has been reached. The concentration is taken across the center of the precipitate, as shown by the white line in the insert. The concentration for all cases are similar, especially as the interface width is increased, and is highlighted by the high resolution of the $\mathrm{y}$-axis. 

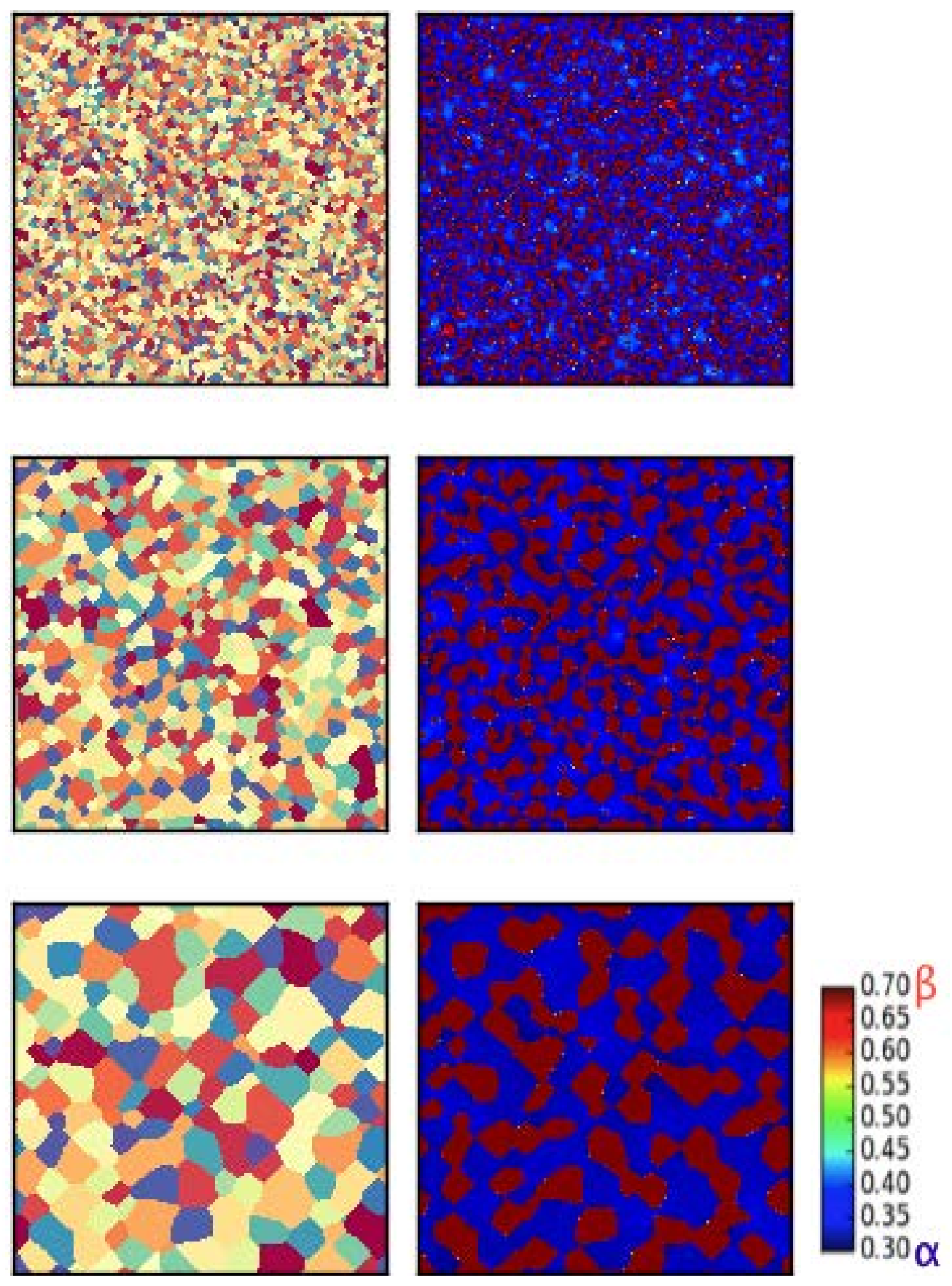

Figure 2: Microstructural evolution showing grain growth on the left, and concentration evolution and coarsening of the $\alpha$ (blue) and $\beta$ (red) phases on the right for the $\mathcal{N}=1$ case. We show the spin and concentration distributions for: (top to bottom) $t=464,4640$ and $46416 M C S$. 


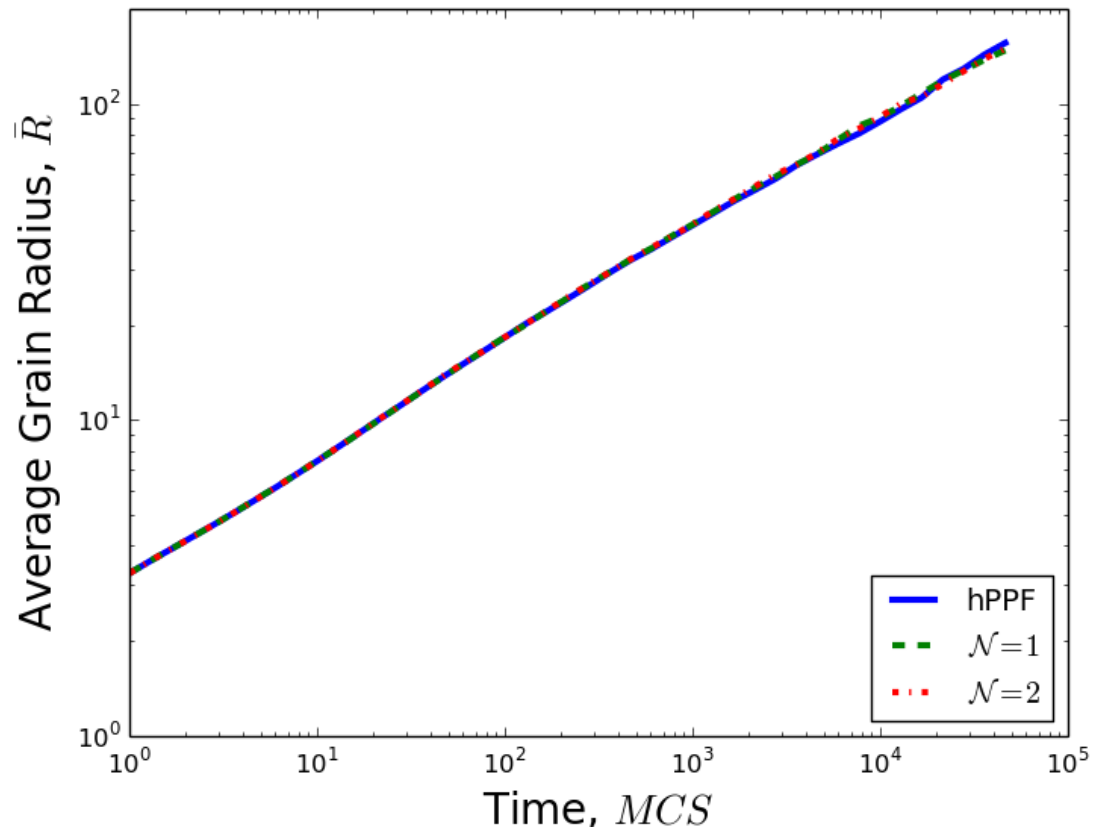

Figure 3: Grain growth curves for hPPF and our simplified model with shells $\mathcal{N}=1$ and 2. We can see that all three curves are virtually identical. 

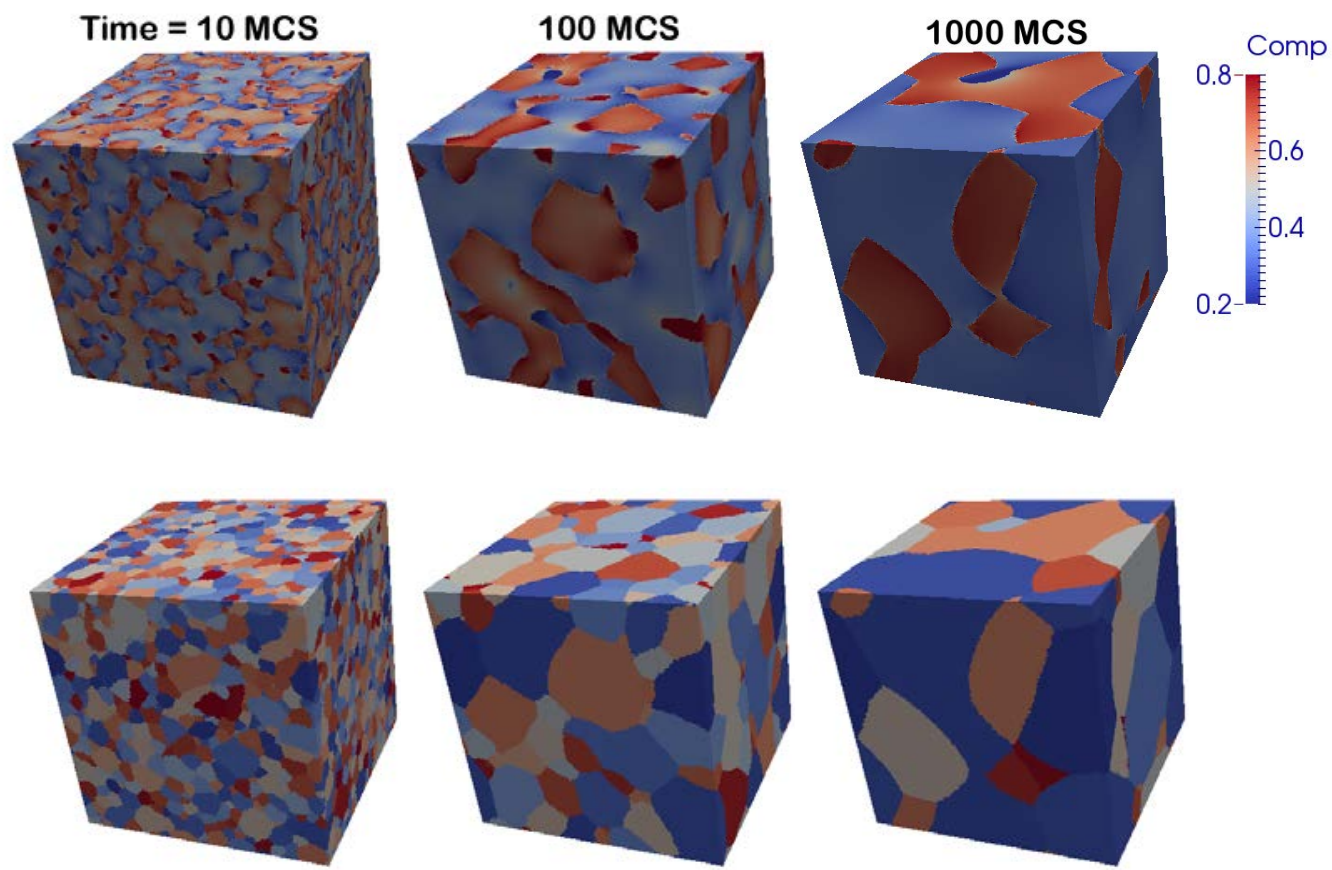

Figure 4: 3D simulation of a polycrystalline microstructural evolution using the discrete curvature definition $\left(\mathcal{N}_{3 D}=1\right)$. The top figures show the concentration evolution, while the bottom shows the microstructural (grain id) evolution. The grain growth exponential was found to be in good agreement with the 2D cases, Table 2. 